\title{
ИЗЛУЧЕНИЕ СВОБОДНО ГРАВИТИРУЮЩЕГО ЗАРЯДА В ПОЛЕ СЛАБОЙ ПЛОСКОЙ ГРАВИТАЦИОННОЙ ВОЛНЫ
}

На основании формулы обобщенного запаздывающего потенциала [1] вычисляется электромагнитное излучение свободно гравитирующего заряда в поле слабой плоской гравитационной волны. Определяются уравнения геодезических и асимптотические выражения для плотности энергии и импульса электромагнитных волн, излучаемых зарядами, движущимися вдоль этих геодезических. Источниками излучения являются не только движущиеся, но и покоящиеся заряды и их кулоновские поля.

\section{1. Введение}

B $\left[{ }^{2}\right]$ нами развит общий метод интегрирования волнового уравнения на фоне неплоской фоновой метрики пространства-времени. В [ $\left.{ }^{1}\right]$ на базе этого метода выведена формула обобщенного запаздывающего потенциала для фоновой метрики в виде слабой плоской гравитационной волны. В данной работе с помощью указанной формулы рассчитывается электромагнитное излучение свободно гравитирующего заряда. Показывается, что мировая линия покоящейся в определенной координатной системе частицы является геодезической, а геодезические для частиц с произвольными начальными скоростями получаются из выражения для мировой линии покоящейся частицы преобразованиями координат, которые представляют собой изометрические преобразования фоновой метрики или несущественно отличаются от последних. Эти преобразования позволяют получать электромагнитное поле движущихся зарядов из электромагнитного поля покоящегося заряда. Принципиальный интерес представляют излучение покоящегося заряда и тот факт, что кулоновское поле заряда становится источником плоских волн.

Проблема, рассматриваемая ниже, частично изучалась обычным методом последовательных приближений в [ $\left.{ }^{3,4}\right]$.

\section{2. Уравнения Максвелла на фоне слабой плоской гравитационной волны и их интегралы}

Рассмотрим монохроматическую плоскую гравитационную волну, распространяющуюся в отрицательном направлении оси * $x$ :

* Единицы измерения выбраны таким образом, чтобы скорость света и гравитационная постоянная были равны единице, 


$$
d s^{2}=d t^{2}-d x^{2}-(1+h) d y^{2}-(1-h) d z^{2},
$$

где

$$
h \equiv h(u)=\alpha \sin \Omega u, \quad u=t+x, \quad \alpha=\text { const }, \quad \Omega=\text { const. }
$$

Предположим, что гравитационная волна - слабая, тогда можем ограничиться членами, линейными относительно $h$ (или параметра $\alpha$ ).

Введем локальный квазиортонормированный изотропный репер ** $\left(l^{\mu}, n^{\mu}, m^{\mu}, \bar{m}^{\mu}\right)$

$$
\begin{aligned}
l^{\mu} & =\frac{1}{\sqrt{2}}(1,-1,0,0), \\
n^{\mu} & =\frac{1}{\sqrt{2}}(1,1,0,0), \\
m^{\mu} & =-\frac{1}{\sqrt{2}}\left(0,0,1-\frac{1}{2} h, i\left(1+\frac{1}{2} h\right)\right) .
\end{aligned}
$$

Электромагнитное поле будем описывать компонентами $\Phi_{A}$ спинора электромагнитного поля, связанными с компонентами тензора электромагнитного поля $F_{\mu \nu}$ следующими соотношениями $\left[{ }^{5}\right]$ :

$$
\begin{aligned}
& \Phi_{0} \equiv F_{\mu v} l^{\mu} m^{v}, \\
& \Phi_{1} \equiv \frac{1}{2} F_{\mu v}\left(l^{\mu} n^{v}+\bar{m}^{\mu} m^{v}\right), \\
& \Phi_{2} \equiv F_{\mu v} \bar{m}^{\mu} n^{v} ; \\
& \frac{1}{2} F_{\mu v}=\Phi_{2} l_{[\mu} m_{v]}+\bar{\Phi}_{2} l_{[\mu} \bar{m}_{v]}+ \\
& \quad+\left(\Phi_{1}+\bar{\Phi}_{1}\right) n_{[\mu} l_{v]}+\left(\Phi_{1}-\bar{\Phi}_{1}\right) m_{[\mu} \bar{m}_{v]}+ \\
& \quad+\Phi_{0} \bar{m}_{[\mu} n_{v]}+\bar{\Phi}_{0} m_{[\mu} n_{v]},
\end{aligned}
$$

где

$$
a_{[\mu} b_{v]} \equiv \frac{1}{2}\left(a_{\mu} b_{v}-a_{v} b_{\mu}\right) .
$$

Уравнения Максвелла на фоне метрики (1) для компонент $\Phi_{A}$

$$
\begin{aligned}
& \Phi_{1,0}-\Phi_{1, x}+\Phi_{0, y}-i \Phi_{0, z}-\frac{1}{2} h\left(\Phi_{0, y}+i \Phi_{0, z}\right)=-\frac{1}{\sqrt{2}} J^{\mu} l_{\mu}, \\
& \Phi_{1,0}+\Phi_{1, x}+\Phi_{2, y}+i \Phi_{2, z}-\frac{1}{2} h\left(\Phi_{2, y}-i \Phi_{2, z}\right)=\frac{1}{\sqrt{2}} J^{\mu} n_{\mu},
\end{aligned}
$$

** Греческие индексы принимают значения $0,1,2,3$, латинские строчные $-1,2$, 3 , латинские прописные $-0,1,2$. Черта над символом функции означает комплексное сопряжение.

*** Запятая обозначает обыкновенную частную производную, $\quad \dot{h}(u) \equiv \frac{d h(u)}{d u}$. 


$$
\begin{aligned}
& \Phi_{0,0}+\Phi_{0, x}+\Phi_{1, y}+i \Phi_{1, z}-\frac{1}{2} h\left(\Phi_{1, y}-i \Phi_{1, z}\right)=\frac{1}{\sqrt{2}} J^{\mu} m_{\mu} \\
& \Phi_{2,0}-\Phi_{2, x}+\Phi_{1, y}-i \Phi_{1, z}-\frac{1}{2} h\left(\Phi_{1, y}+i \Phi_{1, z}\right)+\dot{h} \Phi_{0}=-\frac{1}{\sqrt{2}} J^{\mu} \bar{m}_{\mu} .
\end{aligned}
$$

Здесь $J^{\mu}-$ источник электромагнитного поля. Далее, рассмотрим электромагнитное поле, порожденное одним точечным зарядом $e$, движущимся вдоль геодезической $\xi^{\mu}(t)$

$$
\begin{gathered}
J^{\mu}=\frac{e}{\sqrt{-g}} \frac{d \xi^{\mu}}{d t} \delta(\vec{r}-\vec{\xi}(t)), \\
r^{2} \equiv x^{2}+y^{2}+z^{2} .
\end{gathered}
$$

Отметим, что при метрике (1) имеем $\sqrt{-g}=1+O\left(h^{2}\right)$.

Элиминируя $\Phi_{1}$ из уравнений $(5.1)$ и (5.3), получим скалярное волновое уравнение для $\Phi_{0}$

$$
\begin{aligned}
g^{\mu v} \Phi_{0, \mu v} & \equiv \Phi_{0,00}-\Phi_{0, x x}-(1-h) \Phi_{0, y y}-(1+h) \Phi_{0, z z}= \\
& =J^{\mu}{ }_{, v}\left(m_{\mu} l^{v}-l_{\mu} m^{v}\right) .
\end{aligned}
$$

Конкретизируя метрику в выражении для обобщенного запаздывающего потенциала, приведенного в $\left[{ }^{1}\right]$, можем представить решение уравнения (7) в виде следующего интеграла:

$$
\Phi_{0}\left(x_{0}^{\alpha}\right)=\int J^{\mu}{ }_{, v}\left(m_{\mu} l^{v}-l_{\mu} m^{v}\right) S\left(x^{i}, x_{0}^{i}\right) \delta\left(t-t_{0}+\tau\right) d x d y d z d t,
$$

$$
\begin{gathered}
S\left(x^{i}, x_{0}^{i}\right)=\frac{1}{R}+\frac{\alpha}{2}\left\{\frac{\left(y-y_{0}\right)^{2}-\left(z-z_{0}\right)^{2}}{\left(R-x+x_{0}\right) R^{2}} \sin \left[\Omega\left(t_{0}-R+x\right)\right]+O\left(\frac{1}{R^{2}}\right)\right\} \\
R^{2} \equiv\left(x^{i}-x_{0}^{i}\right)\left(x^{i}-x_{0}^{i}\right) \\
x^{\alpha} \equiv(t, x, y, z) .
\end{gathered}
$$

Отметим, что

$$
t-t_{0}+\tau=0
$$

- уравнение светового конуса. В рассматриваемом нами случае достаточно воспользоваться уравнением светового конуса в приближении плоского пространства-времени и положить $\tau=R$. Чтобы найти интересующее нас решение, необходимо сначала определить мировые линии $\xi^{\mu}(t)$ свободно гравитирующих зарядов и затем вычислить интеграл (8). Подстановка значения $\Phi_{0}$ в уравнения Максвелла (5) даст остальные компоненты $\Phi_{1}, \Phi_{2}$ спинора электромагнитного поля.

\section{3. Мировые линии свободно гравитирующих зарядов}

Предположим, что при отсутствии внешнего электромагнитного поля заряд движется вдоль геодезической, определяемой метрикой (1), т. е. торможение излучением считаем пренебрежимо малым. Покажем, что в этом случае мировая линия покоящегося относительно координатной 
сетки $(x, y, z)$ заряда есть геодезическая, а мировые линии любых движущихся только под действием гравитационного поля (1) зарядов можно вывести из мировой линии покоящегося заряда преобразованиями координат, являющимися или изометрией, т. е. не изменяющими вид метрики (1), или меняющими только частоту $\Omega$ фонового гравитационного излучения. Установление вышеуказанного факта имеет большое практическое значение, так как позволяет получать решение уравнений Максвелла для движущегося заряда из решения уравнений Максвелла для покоящегося заряда преобразованием координат и изменением частоты фонового гравитационного излучения.

Начнем с заряда, покоящегося в начальной точке координатной сетки $(x, y, z)$. Вдоль его мировой линии

$$
\xi^{\mu}(t)=(t, 0,0,0)
$$

имеем $d x^{i}=0$ и по метрике (1) на этой линии $d t=d s, t=t_{1}+s$, где $t_{1}$ - постоянная. Следовательно, $t-t_{1}$ является параметром, равным длине дуги $s$ (собственному времени заряда). Этим же параметром будем пользоваться в любой другой системе координат.

При метрике (1) символы Кристоффеля $\Gamma_{00}^{\mu}$ равны нулю и прямое вычисление показывает, что функции $\xi^{\mu}(s)$ действительно определяют геодезическую.

Проведем преобразование координат

$$
\begin{gathered}
\tilde{t}=\beta(t+v x), \\
\tilde{x}=\beta(v t+x), \\
\tilde{y}=y, \\
\tilde{z}=z ; \\
\beta^{2}=\frac{1}{1-v^{2}}, \quad v=\text { const. }
\end{gathered}
$$

Метрика (1) сохраняет в новых координатах свой общий вид, только $\sin \Omega(t+x)$ заменяется на $\sin \tilde{\Omega}(\tilde{t}+\tilde{x})$, где

$$
\tilde{\Omega}=\sqrt{\frac{1-v}{1+v}} \Omega .
$$

Уравнения геодезической (11) преобразуются в уравнения

$$
\tilde{\xi}^{\mu}=(\beta t, \beta v t, 0,0) .
$$

4-Скорость заряда, движущегося по геодезической (14), имеет вид

$$
\frac{d \tilde{\xi}^{\mu}}{d s}=\frac{d \tilde{\xi}^{\mu}}{d t}=(\beta, \beta v, 0,0) .
$$

В итоге приходим к выводу, что заряд, движущийся с 4-скоростью (15) в поле плоской монохроматической гравитационной волны (1) с частотой $\tilde{\Omega}$, эквивалентен заряду, покоящемуся в поле гравитационной волны (1) с частотой $\Omega=\tilde{\Omega} \sqrt{\frac{1+v}{1-v}}$.

Аналогичная эквивалентность существует между покоящимся и двн- 
жущимся с произвольной скоростью зарядами. Она вытекает из преобразований (12) и следующих изометрических преобразований метрики (1) $\left[{ }^{6}\right]$ :

$$
\begin{aligned}
u^{\prime} & =\tilde{u}, \\
v^{\prime} & =\tilde{v}+b-\operatorname{Re}\left[\left(\tilde{\zeta}+\frac{1}{2} \overline{\tilde{\zeta}} h+\frac{1}{2} g\right) \dot{\bar{g}}-\frac{1}{2}\left(\tilde{\zeta}+\frac{1}{2} g\right) g \dot{h}\right], \\
\zeta^{\prime}=\tilde{\zeta}+g-\frac{1}{2} \bar{g} h, & \\
u & =t+x, \\
v & =-\frac{1}{2}(t-x), \\
\zeta & =y+i z .
\end{aligned}
$$

Здесь $b=$ const, a комплексная функция $g=g(\tilde{u})$ удовлетворяет уравнению

$$
\ddot{g}=\frac{1}{2} \hbar \bar{g},
$$

решение которого дает две произвольные комплексные постоянные: $g(0)$ и $\dot{g}(0)$. Выполняя преобразование координат $(16)$, получим из (14) новые геодезические

$$
\begin{gathered}
u^{\prime}(s)=\sqrt{\frac{1+v}{1-v}}\left(s+t_{1}\right) \\
v^{\prime}(s)=-\frac{1}{2} \sqrt{\frac{1-v}{1+v}}\left(s+t_{1}\right)+b-\frac{1}{2} \operatorname{Re}\left(g \dot{\bar{g}}-\frac{1}{2} g^{2} \dot{h}\right) \\
\zeta^{\prime}(s)=g-\frac{1}{2} \bar{g} h \\
h=\alpha \sin (\tilde{\Omega} \tilde{u})=\alpha \sin \left[\Omega\left(s+t_{1}\right)\right] \\
g=g(\tilde{u})=g\left(\sqrt{\frac{1+v}{1-v}}\left(s+t_{1}\right)\right)
\end{gathered}
$$

Выражения (19) содержат семь произвольных постоянных. Их соответствующий выбор позволяет описать уравнениями (19) все временноподобные геодезические в поле плоской гравитационной волны (1). Так как эти геодезические получены из геодезической покоящейся частицы преобразованием координат, могущим менять только частоту фоновой волны, то излучение заряда, движущегося по геодезической (19), описывается формулами, получаемыми из формул для неподвижного заряда преобразованиями координат (12), (16) и соответствующей заменой частоты гравитационной волны. Вследствие того, что электромагнитное поле в волновой зоне пропорционально малому параметру $\alpha$, достаточно применить преобразование (16) в приближении плоского пространства-времени и взять решение уравнения (18) в виде

$$
g(\tilde{u})=\dot{g}(0) \tilde{u}+g(0) .
$$




\section{4. Излучение неподвижного заряда}

Вычислим интеграл (8) для неподвижного заряда, описываемого следующим специальным видом тока (6):

Имеем

$$
J^{\mu}=(e \delta(\vec{r}), 0,0,0) .
$$

$$
\begin{aligned}
\Phi_{0}\left(x_{0}^{\alpha}\right)=\frac{e}{2} \iint & {\left[\left(1-\frac{1}{2} h\right) \frac{\partial}{\partial y} \delta(\vec{r})+i\left(1+\frac{1}{2} h\right) \frac{\partial}{\partial z} \delta(\vec{r})\right] \times } \\
& \times S\left(x^{i}, x_{0}^{i}\right) \delta\left(t-t_{0}+R\right) d^{3} x d t .
\end{aligned}
$$

Интегрируя сначала по $x^{i}$ (при этом надо учитывать, что аргумент второй $\delta$-функции $t-t_{0}+R\left(x^{i}, x_{0}{ }^{i}\right)$ также зависит от $x^{i}$ !) и затем по $t$, получим следующее асимптотическое выражение:

$\Phi_{0}\left(x^{\alpha}\right)=\frac{e \Omega \alpha}{4 r}\left[\frac{y-i z}{r}-\frac{(y+i z)\left(y^{2}-z^{2}\right)}{r^{2}(r+x)}\right] \cos \Omega(t-r)+O\left(\frac{1}{r^{2}}\right)$.

Из уравнений Максвелла (5) определим остальные компоненты $\Phi_{A}$ :

$$
\begin{gathered}
\Phi_{1}\left(x^{\alpha}\right)=-\frac{e \Omega \alpha}{4 r}\left[\frac{2 i y z}{r(r+x)}-\frac{x\left(y^{2}-z^{2}\right)}{r^{2}(r+x)}\right] \cos \Omega(t-r)+O\left(\frac{1}{r^{2}}\right), \\
\Phi_{2}\left(x^{\alpha}\right)=-\frac{e \Omega \alpha}{4 r}\left[\frac{(y+i z)(r-x)}{r(r+x)}-\frac{(y-i z)\left(y^{2}-z^{2}\right)}{r^{2}(r+x)}\right] \cos \Omega(t-r)+ \\
+\frac{e \Omega \alpha}{2 r} \cdot \frac{y+i z}{r+x} \cos \Omega(t+x)+O\left(\frac{1}{r^{2}}\right)
\end{gathered}
$$

Отметим, что для предотвращения появления расходимости на полуоси $r=-x$ мы добавили к сферическим волнам, исходящим от неподвижного заряда, волну, распространяющуюся в отрицательном направлении оси $x$ :

$$
\begin{aligned}
& \Phi_{0}=\Phi_{1}=0, \\
& \Phi_{2}=\frac{e \Omega \alpha}{2 r} \cdot \frac{y+i z}{r+x} \cos \Omega(t+x)+O\left(\frac{1}{r^{2}}\right) .
\end{aligned}
$$

Последняя волна удовлетворяет с точностью до членов $O\left(\frac{1}{r}\right)$ уравнениям Максвелла (5) для вакуума. Более детальный анализ показывает, что источником этой волны является кулоновское поле, порожденное зарядом $e$.

Определим компоненты $T 0 \alpha \equiv(w, \vec{s})$ тензора энергин-импульса электромагнитного поля, пользуясь соотношениями (2), (4), решением (21) и следующим общим выражением для тензора $T_{\mu \nu}$ :

$$
\begin{gathered}
2 \pi T_{\mu v}=\Phi_{2} \bar{\Phi}_{2} l_{\mu} l_{v}+2 \Phi_{1} \bar{\Phi}_{1} l_{(\mu} n_{v)}-2 \bar{\Phi}_{1} \Phi_{2} l_{(\mu} m_{v)}-2 \Phi_{1} \bar{\Phi}_{2} l_{(\mu} \bar{m}_{v)}+\Phi_{0} \bar{\Phi}_{0} n_{\mu} n_{v}- \\
-2 \bar{\Phi}_{0} \Phi_{1} n_{(\mu} m_{v)}-2 \Phi_{0} \bar{\Phi}_{1} n_{(\mu} \bar{m}_{v)}+\bar{\Phi}_{0} \Phi_{2} m_{\mu} m_{v}+2 \Phi_{1} \bar{\Phi}_{1} m_{(\mu} \bar{m}_{v)}+\Phi_{0} \bar{\Phi}_{2} \bar{m}_{\mu} \bar{m}_{v}, \\
a_{(\mu} b_{v)} \equiv \frac{1}{2}\left(a_{\mu} b_{v}+a_{v} b_{\mu}\right) .
\end{gathered}
$$


В наинизшем неисчезающем приближении имеем следующие асимптотические выражения для плотности энергии $ш$ и потока энергии $\vec{s}$, излучаемых неподвижным на фоне метрики (1) зарядом

$$
\begin{aligned}
w & =\frac{1}{4 \pi}\left(\Phi_{0} \bar{\Phi}_{0}+2 \Phi_{1} \bar{\Phi}_{1}+\Phi_{2} \bar{\Phi}_{2}\right)= \\
& =\frac{e^{2} \alpha^{2} \Omega^{2}}{16 \pi r^{2}}\left\{\frac { 1 - \operatorname { c o s } \vartheta } { 1 + \operatorname { c o s } \vartheta } \left[\cos ^{2} \Omega(t-r)-\right.\right. \\
& \left.-(1-\cos \vartheta) \cos \Omega(t-r) \cos \Omega(t+x)+\cos ^{2} \Omega(t+x)\right]- \\
& -(1-\cos \vartheta)^{2} \cos ^{2} 2 \varphi \cos \Omega(t-r)[\cos \Omega(t-r)- \\
& -\cos \Omega(t+x)]\}+O\left(\frac{1}{r^{3}}\right)
\end{aligned}
$$

$$
\begin{aligned}
s_{x} & =\frac{1}{4 \pi}\left(\Phi_{0} \bar{\Phi}_{0}-\Phi_{2} \bar{\Phi}_{2}\right)= \\
& =\frac{e^{2} \Omega^{2} \alpha^{2}}{16 \pi r^{2}}\left\{\frac{(1-\cos \vartheta) \cos \vartheta}{1+\cos \vartheta}\left[1-\sin ^{2} \vartheta \cos ^{2} 2 \varphi\right] \cos ^{2} \Omega(t-r)+\right. \\
& +\frac{(1-\cos \vartheta)^{2}}{1+\cos \vartheta}\left[1-(1+\cos \vartheta) \cos ^{2} 2 \varphi\right] \cos \Omega(t-r) \cos \Omega(t+x)- \\
& \left.-\frac{1-\cos \vartheta}{1+\cos \vartheta} \cos ^{2} \Omega(t+x)\right\}+O\left(\frac{1}{r^{3}}\right), \\
s_{y} & =\frac{1}{4 \pi}\left[\bar{\Phi}_{1}\left(\Phi_{0}+\Phi_{2}\right)+\Phi_{1}\left(\bar{\Phi}_{0}+\bar{\Phi}_{2}\right)\right]= \\
& =\frac{e^{2} \Omega^{2} \alpha^{2}}{16 \pi r^{2}}\left\{\frac{(1-\cos \vartheta) \sin \vartheta \cos \varphi}{1+\cos \vartheta}\left[1-\sin ^{2} \vartheta \cos ^{2} 2 \varphi\right] \cos ^{2} \Omega(t-r)+\right. \\
& +\frac{(1-\cos \vartheta) \sin \vartheta}{1+\cos \vartheta}\left[\cos 3 \varphi-(1-\cos \vartheta) \cos \varphi \cos ^{2} \varphi\right] \times \\
& \times \cos \Omega(t-r) \cos \Omega(t+x)\}+O\left(\frac{1}{r^{3}}\right),
\end{aligned}
$$

$$
\begin{aligned}
& s_{z}=\frac{i}{4 \pi}\left[\bar{\Phi}_{1}\left(\Phi_{2}-\Phi_{0}\right)-\Phi_{1}\left(\bar{\Phi}_{2}-\bar{\Phi}_{0}\right)\right]= \\
& =\frac{e^{2} \Omega^{2} \alpha^{2}}{16 \pi r^{2}}\left\{\frac{(1-\cos \vartheta) \sin \vartheta \sin \varphi}{1+\cos \vartheta}\left[1-\sin ^{2} \vartheta \cos ^{2} 2 \varphi\right] \cos ^{2} \Omega(t-r)-\right. \\
& -\frac{(1-\cos \vartheta) \sin \vartheta}{1+\cos \vartheta}[\sin 3 \varphi-(1-\cos \vartheta) \sin \varphi \cos 2 \varphi] \times \\
& \times \cos \Omega(t-r) \cos \Omega(t+x)\}+O\left(\frac{1}{r^{3}}\right) \\
& x \equiv r \cos \vartheta \\
& y \equiv r \sin \vartheta \cos \varphi \\
& z \equiv r \sin \vartheta \sin \varphi
\end{aligned}
$$




\section{5. Об излучении движущегося заряда}

Приведем общую схему вычисления излучения заряда, движущегося по произвольной геодезической в поле плоской гравитационной волны (1). С помощью преобразований, обратных преобразованиям (12) и (16), переходим к системе отсчета $x^{\alpha^{\prime}}$, в которой заряд покоится. В новой системе координат компоненты $T^{0^{\prime} \alpha^{\prime}}$ тензора энергии-импульса имеют асимтотический вид $(24),(25)$, только частота $\Omega$ заменяется частотой $\Omega^{\prime}$ :

$$
\Omega^{\prime}=\sqrt{\frac{1+v}{1-v}} \Omega .
$$

С помощью формул $(2),(4),(21),(23)$ можно вычислить и остальные компоненты $T^{i^{\prime} k^{\prime}}$.

Далее, пользуясь преобразованиями (12), (16), переходим обратно к первоначальной системе отсчета $x^{\alpha}$, в которой заряд движется по геодезической. Компоненты тензора энергии-импульса $T^{0 \alpha}\left(x^{\beta}\right)=$ $=\frac{\partial t}{\partial x^{\sigma^{\prime}}} \frac{\partial x^{\alpha}}{\partial x^{\rho^{\prime}}} T^{\sigma^{\prime} \rho^{\prime}}\left(x^{\beta^{\prime}}\right)$ опишут искомые распределения плотности и потока энергии.

В качестве примера приведем асимптотическое выражение электромагнитной энергии, излучаемой зарядом, имеющим на фоне метрики (1) 4-скорость $(\beta, \beta v, 0,0)$. Вычисляя спиноры $\Phi_{A}^{\prime}$ и тензор энергииимпульса $T^{\alpha^{\prime} \beta^{\prime}}$ в системе координат $x^{\alpha^{\prime}}$, в которой заряд покоится, и переходя обратно к первоначальной системе координат $x^{\alpha}$, имеем:

$$
\begin{aligned}
& w=T^{00}=\beta^{2}\left(T^{0^{\prime} 0^{\prime}}+2 v T^{0^{\prime} 1^{\prime}}+v^{2} T^{1^{\prime} 1^{\prime}}\right)= \\
& =\frac{e^{2} \alpha^{2} \Omega^{\prime 2}}{16 \pi r^{\prime 2}}\left\{\frac{r^{\prime}-x^{\prime}}{r^{\prime}+x^{\prime}}\left[1-\left(1-\frac{x^{\prime 2}}{r^{\prime 2}}\right)\left(\frac{y^{2}-z^{2}}{y^{2}+z^{2}}\right)^{2}\right] \frac{\left(r^{\prime}+v x^{\prime}\right)^{2}}{r^{\prime 2}\left(1-v^{2}\right)} \cos ^{2} \Omega^{\prime}\left(t^{\prime}-r^{\prime}\right)-\right. \\
& -\frac{\left(r^{\prime}-x^{\prime}\right)^{2}}{r^{\prime}\left(r^{\prime}+x^{\prime}\right)}\left[1-\left(1+\frac{x^{\prime}}{r^{\prime}}\right)\left(\frac{y^{2}-z^{2}}{y^{2}+z^{2}}\right)^{2}\right] \frac{1-v}{1+v} \cos \Omega^{\prime}\left(t^{\prime}-r^{\prime}\right) \cos \Omega(t+x)+ \\
& \left.+\frac{r^{\prime}-x^{\prime}}{r^{\prime}+x^{\prime}} \cdot \frac{1-v}{1+v} \cos ^{2} \Omega(t+x)\right\}, \\
& t^{\prime}=\beta(t-v x), \\
& x^{\prime}=\beta(-v t+x) \text {, } \\
& r^{\prime 2}=\beta^{2}(x-v t)^{2} f y^{2}+z^{2}, \\
& \Omega^{\prime}=\sqrt{\frac{1+v}{1-v}} \Omega .
\end{aligned}
$$

Видим, что в ультрарелятивистском пределе $v \rightarrow 1$ энергия $w$ и частота излучения $\Omega^{\prime}$ быстро возрастают.

\section{ЛИТ Е Р А Т У Р А}

1. У н т В., Изв. АН ЭССР, Физ. Матем., 27, 132 (1978).

2. Unt, V., Kuusk, P., ENSV TA Toimet., Füüs. Matem., 25, 234 (1976).

3. Д и м а ншт ей н Ф. А., Укр. физ. ж., 16, 1877 (1971). 
4. Гин з бург В. Л., Цы тович В. Н., Изв. ВУЗов, Радиофизика, 18, № 2, 173 (1975).

5. N ew ma n, E. T., Pe nrose, R., J. Math. Phys., 3, 566 (1962); 4, 998 (1963).

6. Ehlers, J., Kundt, W., In: Gravitation: An Introduction to Current Research, ed. by L. Witten, New York, London, 1962.

$\begin{array}{cc}\text { Институт физики } & \text { Поступила в редакцию } \\ \text { Академии наук Әстонской } & \text { ССР } 1977\end{array}$

Piret KUUSK

\section{VABALT GRAVITEERUVA LAENGU KIIRGUS NÕRGA TASASE GRAVITATSIOONILAINE VÄLJAS}

Oldistatud retardeeritud potentsiaali valemi $\left.{ }^{[1}\right]$ abil on arvutatud vabalt graviteeruva laengu väli nõrga tasase gravitatsioonilaine taustal. On leitud geodeetiliste joonte võrrandid ning piki neid jooni liikuvate laengute kiiratud elektromagnetvälja energia ja impulsi asümptootilised avaldised. Kiirguse allikaks ei ole ainult liikuvad laengud, vaid ka paigalseisvad laengud ja nende Coulomb'i väljad.

\section{Piret KUUSK}

\section{RADIATION OF A FREELY GRAVITATING CHARGE IN THE FIELD OF A WEAK PLANE GRAVITATIONAL WAVE}

The radiation of a freely gravitating electric charge in the field of a weak plane gravitational wave is calculated by using the integral representation of the solution of a generalized wave equation [ $\left.{ }^{1}\right]$. Asymptotic expressions for the energy and momentum of the electromagnetic field are found. It turns out that, besides moving charges, a charge at rest and its Coulomb field are the sources of radiation. 